\title{
ILMENITAS KIMBERLITICAS DA MINA DE DIAMANTES DE ROMARIA, MG
}

\author{
DARCY P. SVISERO* E HENRY O.A. MEYER**
}

\begin{abstract}
Magnesium ilmenites have been found in the diamond bearing conglomerate croping out the city of Romaria, Minas Gerais State. The ilmenites are associated with other heavy minerals, including magnetite, hematite, limonite, staurolite, zircon, pyrope knorringite and almandine grossular garnets, tourmaline, epidote, kyanite, monazite, corundum, anatase, hornblende, and rutile as well as diamond.

Microprobe data (29 analyses) indicate that the ilmenites have $\mathrm{MgO}$ and $\mathrm{Cr}_{2} \mathrm{O}_{3}$ ranging from 7.0 to 12.5 and from 0 to $3.5 \%$, respectively, and contents of $\mathrm{Al}_{2} \mathrm{O}_{3}, \mathrm{CaO}$ and $\mathrm{MnO}$ usually lower than $0,5 \%$. Thus, the Romaria ilmenites are similar in chemistry to ilmenites of kimberlite concentrates and, also, to ilmenites of ultramafic xenoliths from kimberlites of worldwide localities. On the other hand, physical characteristics of the ilmenites and other as* sociated kimberlitic minerals clearly suggest the presence of diamondiferous kimberlites nearby Romaria.
\end{abstract}

INTRODUÇÃo Análises químicas è mineralógicas revelaram a presença de ilmenitas magnesianas nos concentrados da mina de diamantes de Romaria, localizada nas proximidades da cidade de Romaria, Minas Gerais (Fig. 1).

É fato bem conhecido que ilmenitas magnesianas são um dos minerais mais típicos de kimberlitos, constituindo-se, por conseqüência, em um dos mais importantes guias de prospecção dessas rochas. Pela alteração intempérica, alguns de seus minerais são destruídos, especialmente a olivina, enquanto outras fases mais resistentes, entre as quais se incluem a ilmenita magnesiana e a granada piropo-knorringita, são arrastadas pelas drenagens próximas. Rastreamento sistemático dessas fases constitui um dos métodos mais utilizados e eficientes na prospecção de kimberlitos.

A ocorrência de diamante nas imediações de Romaria, conhecida desde a segunda metade do século passado (Leonardos, 1956), motivou o aparecimento de um grande número de garimpos, de início rudimentares a semimecanizados, que evoluíram mais tarde para as instalações mecanizadas operadas atualmente pela Extratífera de Diamantes do Brasil S.A. (Exdibra).

Neste trabalho, são apresentadas análises químicas quantitativas de ilmenitas associadas ao conglomerado lavrado em Romaria. É discutido o significado dessas ilmenitas enriquecidas em magnésio e cromo, e sua relação com os kimberlitos dispersores do diamante da região de Romaria.

CONSIDERAÇÕES SOBRE A GEOLOGIA LOCAL A mina de diamantes de Romaria, antiga Água Suja, situa-se nas proximidades da cidade de Romaria (MG), localizada nas cabeceiras do Rio Bagagem, borda norte-nordeste da Bacia do Paraná.

Os primeiros trabalhos de geologia sobre a Mina de Romaria são devidos a Campos (1891), Hussak (1891 e 1894), Calógeras (1895), Porcheron (1903) e Draper (1911), e versaram sobre as formas de ocorrência e aspectos gerais da lavra de diamante no local. Informações ge-

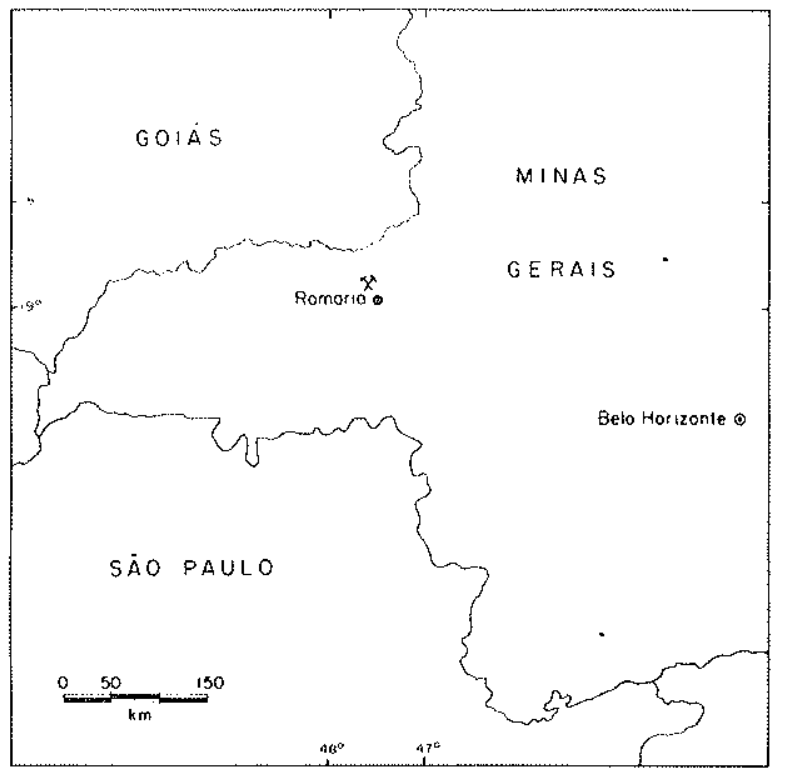

Figura 1 - Localização geográfica da mina de diamantes de Romaria

rais sobre a geologia da região encontram-se nos trabaIhos de Leonardos (1956), Hasui (1968 e 1969) e Barbosa et al. (1970).

Afloram na região micaxistos do Grupo Araxá, constituidos essencialmente por quartzo e micas, cortados localmente por veios pegmatóides de composição granítica. Corpos de metabasito concordantes com a xistosidade regional N-SE são extremamente comuns em toda região (Barbosa et al., 1970). Nos contatos entre os micaxistos e os metabasitos, são frequientes veios de turmalinito formados por um agregado fino de cristais de turmalina negra.

Sobre esse embasamento antigo, assentam arenitos avermelhados da Formação Botucatu, que localmente se

\footnotetext{
* Instituio de Geociências, Universidade de São Paulo, Caixa Postal 20899, CEP 01000, São Paulo, SP

** Geosciences Department, Purdue University, West Lafayette, Indiana 47.907, EUA
} 
apresentam bem cristalizados, seguidos pelo pacote vulcanoclástico da Formaçăo Uberaba (Hasui, 1968). A Formação Uberaba, de idade cretácea, é formada essencialmente por arenitos finos de coloração verde-clara, contendo intercalações de conglomerados de espessura e natureza diversas. O conglomerado basal diamantífero possui espessura entre 2 e $6 \mathrm{~m}$, e uma cobertura argilosa rica de concreções ferruginosas, que, na área, varia de 10 a $30 \mathrm{~m}$ (Svisero et. al., 1981).

Diversos trabalhos efetuados nos últimos anos ampliaram consideravelmente o conhecimento da geologia e do diamante da Mina de Romaria. Suguio et al. (1979) mostraram que o conglomerado diamantífero lavrado em Romaria foi depositado na forma de leques aluviais originados em climas secos. Segundo esses autores, os sedimentos foram transportados por fluxos viscosos de detritos e corridas de lama e depositados nas irregularidades do embasamento local constituido por micaxistos do Grupo Araxá e arenitos da Formação Botucatu. Svisero (1979) identificou granadas tipicamente kimberliticas, formadas pelas moléculas piropo $\mathrm{Mg}_{3} \mathrm{Al}_{2}\left(\mathrm{SiO}_{4}\right)_{3}$ e knorringita $\mathrm{Mg}_{3} \mathrm{Cr}_{2}\left(\mathrm{SiO}_{4}\right)_{3}$, nos concentrados provenientes da lavagem do conglomerado. Posteriormente, Svisero et al. (1980) estenderam esses estudos aos demais constituintes dos concentrados, relacionando a origem dos minerais pesados com as variedades litológicas da região. Mais recentemente, Svisero et al. (1981) mapearam com detalhe a área de concessão da Exdibra. Ainda nessa oportunidade, esses últimos autores caracterizaram sob diversos pontos de vista - cor, morfologia, defeitos, granulometria, etc. - o diamante de Romaria e discutiram aspectos gerais relacionados à lavra do diamante no Brasil. Desses trabalhos emergiram diversas evidências, apontando para a existência de intrusões kimberlíticas nas proximidades da Mina de Romaria.

Levantamentos geofísicos executados nos últimos anos na parte oeste de Minas Gerais indicaram a existência de um grande número de anomalias magnetométricas na região de Romaria e Estrela do Sul. Svisero et al. (1979) verificaram que algumas dessas anomalias correspondem a corpos anelares de dimensões reduzidas, que poderiam corresponder aos kimberlitos dispersores do diamante da região.
MATERIAL E TÉCNICAS DE ESTUDO As ilmenitas analisadas fazem parte da assembléia de minerais pesados associados ao conglomerado basal da Formação Uberaba (Hasui, 1968), constituído por blocos centimétricos a decimétricos de micaxistos, metabasitos, filitos, basaltos e arenitos, dispersos em uma matriz argilo arenosa de coloração avermelhada (Svisero et al., 1981). O conglomerado contém ainda grande quantidade de minerais pesados distribuídos de forma irregular na matriz, entre os quais se incluem, além da própria ilmenita, magnetita, hematita, concreções limoníticas, estaurolita, turmalina, zircão, rutílio, epídoto, hornblenda, cianita, monazita, coríndon, granadas piropo-knorringita e almandina grossulária, e diamante (Svisero et al., 1980).

Os concentrados provenientes da lavagem e da concentração do conglomerado são compostos essencialmen te de opacos (de $95 \%$ a $99 \%$ ), entre estes predominando, de forma absoluta, magnetita e concreções limoníticas. As ilmenitas foram isoladas removendo-se os grãos magnéticos com auxílio de ímã manual. Como características gerais, os fragmentos de ilmenita apresentam dimensões entre 1 e $8 \mathrm{~mm}$, brilho submetálico, contornos angulosos e ausência de intercrescimentos e de alteração superficial.

As ilmenitas foram inicialmente identificadas por meio de diagramas de raios $\mathrm{X}$ obtidos pelo método fotográfico, utilizando-se câmara de Gandolfi de 114,59 mm de diâmetro. A seguir, os grãos foram montados em epóxi e analisados em uma microssonda automática, modelo MAC-500, no Departamento de Geociências da Universidade Purdue, dos Estados Unidos. Informações referentes à programação analítica desen volvida podem ser encontradas em Svisero et al. (1977).

DISCUSSÃo Ilmenitas kimberliticas distinguem-se dos demais tipos por conter, respectivamente, teores de $\mathrm{MgO}$ e de $\mathrm{Cr}_{2} \mathrm{O}_{3}$ variando entre $4,0 \%$ e $23,0 \%$ e de $0 \%$ a 8,0\% (Frantesesson, 1970; Gurney et al., 1973; e Sobolev, 1977). Essas características estão evidenciadas na Tab. 1, que mostra algumas análises de ilmenitas de kimberlitos da África do Sul, Lesoto, Estados Unidos, Canadá, Brasil, União Soviética, Índia e Austrália.

Tabela 1 - Análises químicas representativas das ilmenitas de Romaria, $M G$

\begin{tabular}{|c|c|c|c|c|c|c|c|c|c|c|c|c|}
\hline & $\mathrm{R}[-0]$ & RI-04 & RI-08 & RI.09 & R I-12 & $121-17$ & $\mathrm{Rl}-20$ & $\mathrm{R} \mid-21$ & $\mathrm{R} \mid-22$ & $\mathrm{R} 1-23$ & RI- 28 & $\mathrm{RI}-29$ \\
\hline $\mathrm{TiO}_{2}$ & 52,77 & 52,40 & 51,65 & 51,50 & 53,20 & 53,09 & 50,78 & 52.57 & 51,70 & 53,13 & $\$ 2,17$ & 52,75 \\
\hline $\mathrm{Al}_{2} \mathrm{O}_{3}$ & 0,13 & 0,20 & 0,20 & 0,29 & 0,34 & 0,30 & 0,09 & 0,27 & 0,25 & 0,36 & 0,23 & $0,21^{\prime}$ \\
\hline $\mathrm{Cr}_{2} \mathrm{O}_{3}$ & 1,15 & 0,20 & 0,08 & 1,46 & 1,71 & 2,77 & 1,16 & 0,02 & 1,72 & 3,32 & 2,56 & 1,39 \\
\hline $\mathrm{FeO}$ & 35,73 & 36,99 & 38,25 & 35,82 & 32,82 & 32,71 & 41,13 & 36,17 & 33,63 & 30,96 & 34,54 & 33,13 \\
\hline $\mathrm{MgO}$ & 10,42 & 9,87 & 9,42 & 10,34 & 12,07 & 11,66 & 7,83 & 11,14 & 11,43 & 12,07 & 11,11 & $\pi 1,95$ \\
\hline $\mathrm{CaO}$ & 0,03 & 0,12 & 0,09 & 0,07 & 0,03 & 0,05 & 0,00 & 0,04 & 0,10 & 0,00 & 0,03 & 0,03 \\
\hline $\mathrm{MnO}$ & 0,45 & 0,38 & 0,53 & 0,33 & 0,32 & 0,28 & 0,34 & 0,34 & 0,32 & 0,16 & 0,31 & 0,32 \\
\hline Total & 100,67 & 100,15 & 100,21 & 99,72 & 100,50 & 100,76 & 101,34 & 100,54 & 99,16 & 300,00 & 100,95 & 99,79 \\
\hline $\mathrm{Ti}$ & 0,917 & 0,918 & 0,907 & 0,902 & 0,915 & 0,913 & 0,893 & 0,909 & 0,904 & 0,918 & 0,900 & 0,914 \\
\hline AT & 0,003 & 0,004 & 0,004 & 0,007 & 0,008 & 0,007 & 0,002 & 0,006 & 0,006 & 0,009 & 0,006 & 0,0015 \\
\hline $\mathrm{Cr}$ & 0,020 & 0,003 & 0,001 & 0,026 & 0,031 & 0,049 & 0,021 & 0,000 & 0,031 & 0,060 & 0,045 & 0,025 \\
\hline $\mathrm{Fe}^{3+}$ & 0,140 & 0,154 & 0,180 & 0,164 & 0,128 & 0,114 & 0,190 & 0,172 & 0,156 & 0,088 & 0,148 & 0,138 \\
\hline $\mathrm{Fe}^{2+}$ & 0,551 & 0,566 & 0,567 & 0,533 & 0,499 & 0,511 & 0,614 & 0,524 & 0,497 & 0,507 & 0,514 & 0,500 \\
\hline $\mathrm{Mg}$ & 0,359 & 0,342 & 0,327 & 0,358 & 0,411 & 0,397 & 0,272 & 0,381 & 0,396 & 0,414 & 0,379 & 0,410 \\
\hline$\overline{C a}$ & 0,000 & 0,002 & 0,002 & 0,001 & 0,000 & $0,000)$ & 0,000 & 0,000 & 0,382 & 0,000 & 0,000 & 0,000 \\
\hline $\mathrm{M} 13$ & 0,008 & 0,007 & 0,010 & 0,006 & 0,006 & 0,005 & 0,006 & 0,006 & 0,006 & 0,002 & 0,006 & 0,006 \\
\hline $\mathrm{O}$ & 2,930 & 2,923 & 2,910 & 2,918 & 2,936 & 2,943 & 2,905 & 2,914 & 2,922 & 2,956 & 2,926 & 2,931 \\
\hline $\mathrm{MgSiO}_{3}$ & 31,43 & 29,61 & 28,26 & 31,02 & 36,21 & 35,98 & 23,49 & 33,42 & 34,29 & 36,21 & 33,33 & 35,85 \\
\hline FeTiO, & 60,48 & 61,39 & 61,29 & 57,82 & 55,14 & 56,55 & 66,31 & 57,51 & 54,04 & 55,69 & 56.62 & $\$ 4,80$ \\
\hline $\mathrm{Fe}_{2} \mathrm{O}_{3}$ & 8,08 & 8,79 & 10,24 & 9,36 & 7,44 & 6,63 & 10,80 & 9,93 & 8,93 & 5,09 & 8,57 & 7,96 \\
\hline
\end{tabular}


Tabela 2 - Análises químicas representativas de ilmenitas constituintes de concentrados de kimberlitos e de xenólitos ultramáficos de Kimberitos

\begin{tabular}{|c|c|c|c|c|c|c|c|c|c|c|c|c|}
\hline & 01 & 02 & 03 & 04 & os & 06 & 07 & 08 & 09 & 10 & 11 & 12 \\
\hline $\mathrm{SiO}_{2}$ & - & - & 0,07 & 0,01 & - & - & 0,24 & 0,09 & 0,07 & 0,15 & 0,38 & - \\
\hline $\mathrm{TiO}_{2}$ & $54,6 !$ & 49,48 & 49,42 & 52,12 & 48,0 & 54,39 & 53,37 & 51,0 & 54,1 & 39,5 & 51,2 & 50,88 \\
\hline $\mathrm{Al}_{2} \mathrm{O}_{3}$ & 0,34 & 0,18 & 0.63 & 0,34 & - & 0,51 & 0,30 & 0,08 & 0,46 & 0,67 & 0,57 & 0,23 \\
\hline $\mathrm{Cr}_{2} \mathrm{O}_{3}$ & 2,05 & 0.92 & 0.13 & 2,55 & 1,2 & 0,65 & 4,03 & 1,50 & 0,59 & 1,74 & 0,36 & 0,19 \\
\hline $\mathrm{FeO}$ & 21,79 & 28,72 & 29,52 & 15,91 & 27,9 & 25,78 & 23,73 & 30,8 & 22,6 & 33,4 & 26,8 & 40,49 \\
\hline $\mathrm{Fe}_{2} \mathrm{O}_{3}$ & 5,30 & 11,67 & 12,76 & 11,24 & 14,4 & 5,57 & - & 7,99 & 4,50 & 17,3 & 6,5 & - \\
\hline $\mathrm{MgO}$ & 15,14 & 8,72 & 8,25 & 16,99 & 8,4 & 12,76 & 16,43 & 8,66 & 11,40 & 6,12 & 12,1 & 7,84 \\
\hline $\mathrm{CaO}$ & - & - & 0,05 & 0,01 & - & 0,04 & 0,34 & 0,02 & 0,02 & 0,00 & 0,00 & 0,08 \\
\hline $\mathrm{MnO}$ & - & - & 0,24 & 0,66 & 0,2 & 0,25 & 0,54 & 0,28 & 0,27 & 0,22 & 0,34 & 0,30 \\
\hline $\mathrm{NiO}$ & 0,36 & 0,24 & - & - & - & - & - & - & س. & $\ldots-$ & - & - \\
\hline Tolal & 99,54 & 99,70 & 101,02 & 99,81 & 100,1 & 100,05 & 99,04 & 99,6 & 99,5 & 99,1 & 98,2 & $100,0 t$ \\
\hline $\mathrm{MgTiO}_{3}$ & 45,42 & 26,36 & 24,75 & 50,97 & 25,2 & 38,58 & - & 26,0 & 34,2 & 18,4 & 36,3 & - \\
\hline $\mathrm{FeTiO} 3$ & 46,00 & 60,63 & 62,32 & 33,59 & 58,9 & 54,42 & - & 65,0 & 60,4 & 70,5 & 56,6 & - \\
\hline $\mathrm{Fe}_{2} \mathrm{O}_{3}$ & 5,30 & $1\}, 68$ & 12,76 & 11,24 & 14,4 & 5,57 & - & 7,99 & 4,50 & 17,3 & 6,5 & $-m$ \\
\hline
\end{tabular}

01 e 02 - África do Sul (Mitchell, 1973); 03 e 04 - Lesoto (Boctor, 1980; e Boyd e Nixon, 1973); 05 - Canadá (Mitchell, 1979 ); 06 - Estados Unidos (Boctor e Meyer, 1979); 07 - Índica (Akella et al. 1979); 08 e 09 - Brasil (Svisero et al., 1977); 10 e 11 - União Soviética (Sobolev, 1977); 12 - Austrália (Ferguson e Sheraton 1979)

Entretanto, embora características, as ilmenitas mag. nesianas não são exclusivas de kimberlitos, sendo encontradas também em carbonatitos (Bergst $\phi 1,1972$ ). Analisando ilmenitas do carbonatito de Jacupiranga, Boctor e Svisero (1978) encontraram teores de MgO variando entre $12,0 \%$ e $15,0 \%$. Trata-se de valores excepcionalmente altos para carbonatitos, cujas ilmenitas possuem, em geral, menos de 5,0\% de $\mathrm{MgO}$. A distinção entre esses dois tipos de ilmenita baseia-se em outras impurezas, especialmente $\mathrm{Cr}_{2} \mathrm{O}_{3}$ e $\mathrm{MnO}$. Ilmenitas de kimberlitos, em geral cromíferas, são pobres em $\mathrm{MnO}$, cujos teores raramente alcançam $0,5 \%$. No caso dos carbonatitos,, 0 comportamento é inverso; suas ilmenitas contêm teores de $\mathrm{MnO}$ variáveis entre $3,0 \%$ e $5,0 \%$, e concentrações de $\mathrm{Cr}_{2} \mathrm{O}_{3}$ menores de 0,05\% (Boctor e Svisero, 1978).

De um modo geral, as ilmenitas magnesianas são encontradas nos kimberlitos sob três formas distintas: a) como grãos irregulares possuindo dimensões entre $1 \mathrm{e}$ $5 \mathrm{~mm}$, e representando parte da massa fundamental dessas rochas; $b$ ) sob a forma de xenocristais (cristais isolados de ilmenita) e de xenólitos discretos (agregados de cristais de ilmenita) de formas ovalóides e dimensões variáveis entre 0,5 e $10 \mathrm{~cm}$; e c) como intercrescimentos com olivina, enstatita, diopsídio, piropo, flogopita e zircão, podendo o conjunto alcançar dimensões de até $20 \mathrm{~cm}$ de diâmetro (Boyd e Nixon, 1973; Sobolev et al., 1975; e Meyer, 1977). Mais raramente, as ilmenitas ocorrem sob a forma de inclusões microscópicas em diamantes naturais (Meyer e Svisero, 1975).

Tendo em vista as características das amostras estudadas - grãos monominerálicos aparentemente homogêneos de dimensões entre 1 e $8 \mathrm{~mm}-$, as ilmenitas de Romaria devem ter constituído originalmente, ao que tudo indica, xenocristais e/ou parte da massa fundamental do kimberlito.

A Tab. 2 reúne doze análises químicas quantitativas de ilmenitas de Romaria, representativas de um total de 29 análises executadas. Em linhas gerais, as ilmenitas estudadas apresentam caracteristicas típicas de ilmenitas de kimberlitos, contendo teores de $\mathrm{MgO}$ entre 7,0\% e 12,5\% e valores de $\mathrm{Cr}_{2} \mathrm{O}_{3}$ entre $0 \%$ e $3,5 \%$. Outros óxidos, como $\mathrm{Al}_{2} \mathrm{O}_{3}, \mathrm{CaO}$ e $\mathrm{MnO}$, representam pequenas impurezas com teores inferiores a $0,5 \%$. Segundo Lovering e

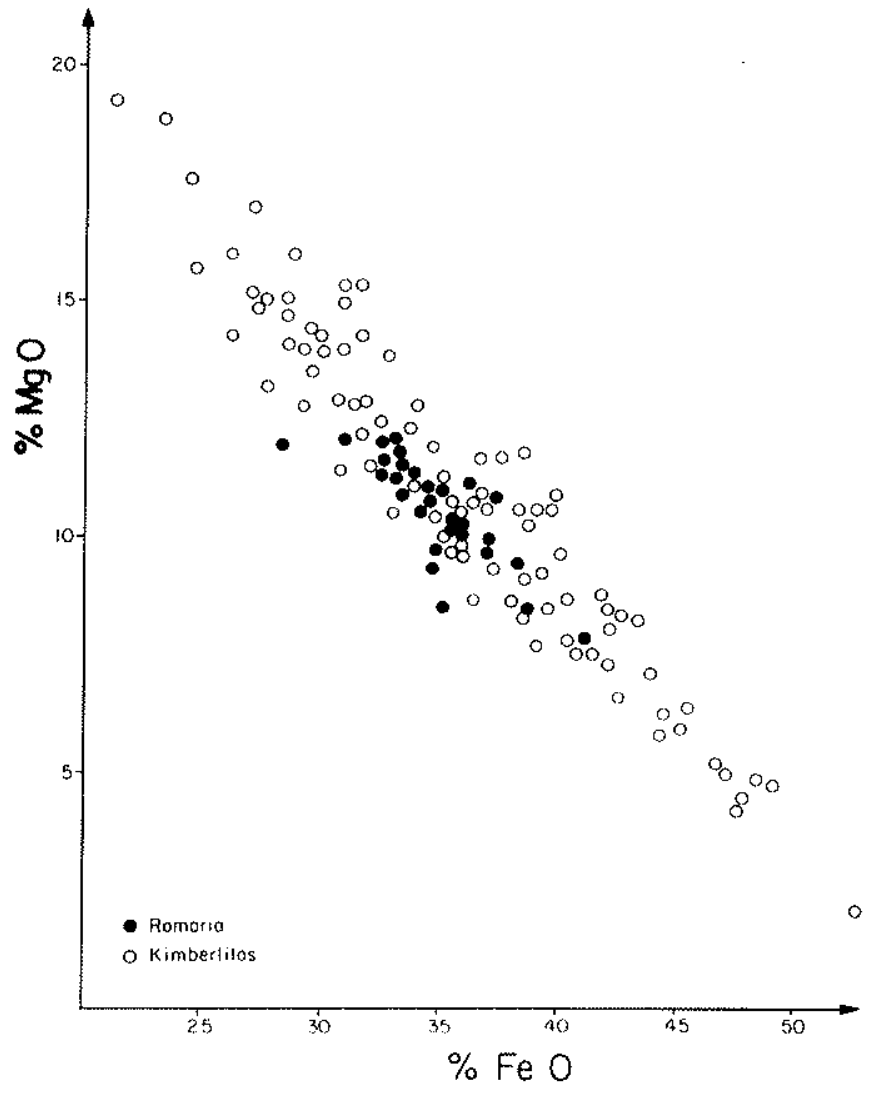

Figura 2 - Variação da relação $\mathrm{MgO}-\mathrm{FeO}$ de ilmenitas de $\mathrm{Ro}$. maria comparadas com ilmenitas de kimberlitos da África do Sul, Brasil, Canadá, Estados Unidos, Lesoto e União Soviética. Dados comparativos extraidos de Mitchell (1973), Boyd e Nixon (1973), Gurney et al. (1973), McCallister et al. (1975), Sobolev (1977), Svisero et al. (1977), Boctor e Meyer (1979), Ferguson e Sheraton (1979), Mitchell (1979) e Boctor e Boyd (1980)

Widdowson (1968), o enriquecimento em magnésio por parte das ilmenitas é um indicador de valores altos para a razão $\mathrm{Mg} / \mathrm{Mg}+\mathrm{Fe}^{2+}$ da rocha original, onde teve lugar a formação desses minerais. 
A Fig. 2 reúne as concentraçōes de $\mathrm{MgO}$ e $\mathrm{FeO}$ enquanto a Fig. 3 corresponde ao diagrama ternário, expresso em termo das moléculas $\mathrm{MgTiO}_{3}$ (geikielita), Fe$\mathrm{TiO}_{3}$ (ilmenita) e $\mathrm{Fe}_{2} \mathrm{O}_{3}$ (hematita). Ambas as figuras mostram que as ilmenitas de Romaria se situam perfeitamente dentro dos respectivos campos de variação de ilmenitas kimberlíticas, estabelecidos a partir de dados referentes a ilmenitas de kimberlitos da África do Sul, Lesoto, Brasil, Canadá, Estados Unidos, União Soviética e Austrália.

Haggerty (1975) verificou que a proporção de $\mathrm{Fe}_{2} \mathrm{O}_{3}$ em solução sólida acarreta variações de magnetismo nas ilmenitas de kimberlitos. A linha tracejada divide o diagrama da Fig. 3 em duas regiões distintas, mostrando que valores de $\mathrm{Fe}_{2} \mathrm{O}_{3}$ acima de $25 \%$ tornam as ilmenitas magnéticas. As amostras de Romaria situam-se dentro do campo das ilmenitas desprovidas de magnetismo, confirmando observações efetuadas durante a separação dos demais opacos dos concentrados estudados.

A variação em cromo exibida pelas ilmenitas de Romaria está ilustrada na Fig. 4, relacionando os valores de $\mathrm{Cr}^{3+} \mathrm{e} \mathrm{Fe}^{3+}$ entre ilmenitas kimberlíticas em geral. Observa-se pela referida figura que a correlação entre esses dois elementos não está bem definida, fato já notado anteriormente por Boyd e Nixon (1973). Entretanto, correlacionando valores de $\mathrm{Cr}_{2} \mathrm{O}_{3}$ e $\mathrm{MgO}$ de ilmenitas kimberlíticas, Haggerty (1975) obteve uma variação aparentemente parabólica entre esses dois óxidos. Nixon e Kresten (1973) tentaram caracterizar kimberlitos de Lesoto e África do Sul a partir das relações $\mathrm{Cr} / \mathrm{Ni}$ de suas ilmenitas, sem contudo obter resultados conclusivos. $O$ que parece certo a respeito do cromo é que a presença desse elemento nas ilmenitas kimberlíticas indica a atuação de pressões elevadas durante a cristalização desse mineral (Boyd e Nixon, 1973).

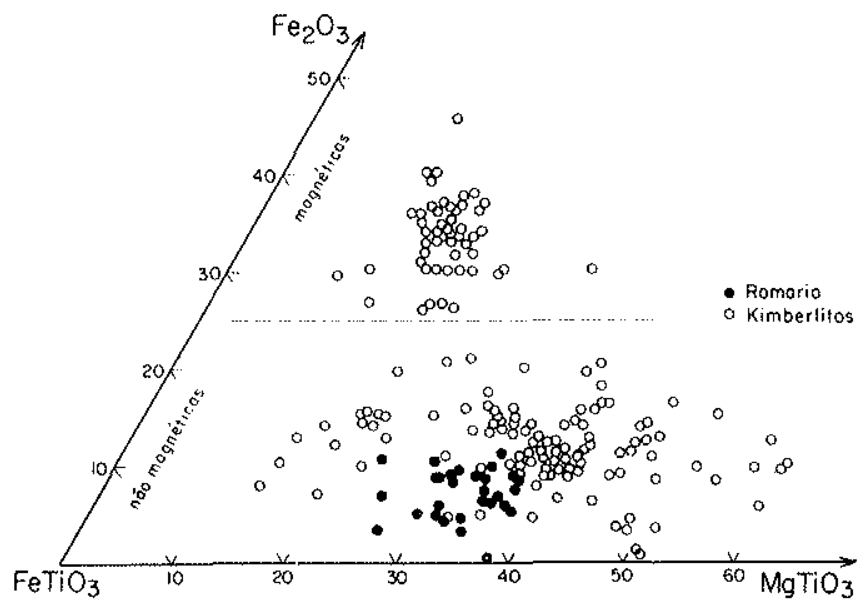

Figura 3 - Variação das moléculas $\mathrm{FeTiO}_{3}$ (ilmenita), $\mathrm{MgTiO}_{3}$ (geikielita) e $\mathrm{Fe}_{2} \mathrm{O}_{3}$ (hematita) de ilmenitas de Romaria compa" radas com ilmenitas de kimberlitos da Africa do Sul, Brasil, Canadá, Estados Unidos, Lesoto e Uniao Soviética. Dados comparativos extraidos de Mitchell (1973), Boyd e Nixon (1973), Hag. gerty (1975), McCallister et al. (1975), Smith et al. (1976), Svisero et al. (1977), Mitchell (1979) e Boctor e Boyd (1980)

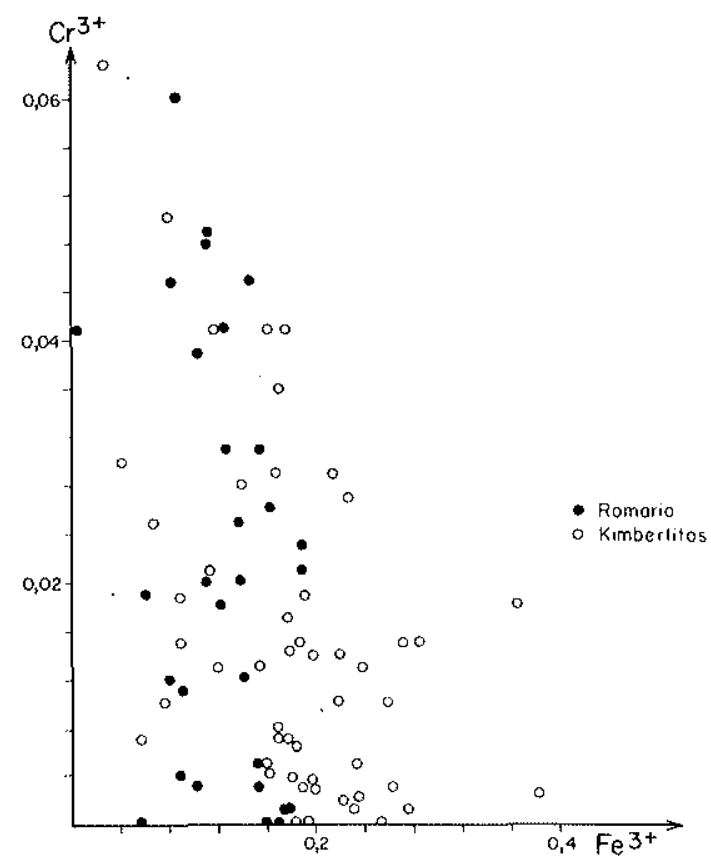

Figura 4 - Variação da relação $\mathrm{Cr}^{3+}-\mathrm{Fe}^{3+}$ de ilmenitas de $\mathrm{Ro}$ maria comparadas com ilmenitas de kimberlitos da África do Sul, Brasil, Lesoto e União Soviética. Dados comparativos ex traidos de Nixon e Boyd (1973), Svisero et al. (1977), Sobolev (1977) e Pasteris et al. (1979)

CONCLUSÕES FINAIS Ilmenitas magnesianas com teores de $\mathrm{MgO}$ entre 7,0\% e 12,5\% ocorrem entre os constituintes dos concentrados provenientes da lavagem mecanizada do conglomerado basal da Formação Uberaba, operada nas instalações de Exdibra S.A., situada nas imediações da cidade de Romaria. Além de altos teores em $\mathrm{MgO}$, essas ilmenitas contêm também de $0 \%$ a $3,5 \%$ de $\mathrm{Cr}_{2} \mathrm{O}_{3}$, ao lado de concentraçōes menores de $\mathrm{MnO}$ $(0,5 \%)$, indicando que elas são realmente de natureza kimberlítica.

De um modo geral, os fragmentos de ilmenita apresentam-se angulosos, homogêneos e quase isentos de alteração, concentrando-se no intervalo de granulação de 1 a $8 \mathrm{~mm}$. Essas características indicam, em princípio, que os kimberlitos dispersores se situam próximos à área de ocorrência atual dos conglomerados. Essa hipótese é corroborada pelas características dos demais minerais kimberlíticos presentes nos concentrados - granada piropo cromífera e o próprio diamante - - que exibem feições indicativas de transporte pequeno. As faces e arestas abauladas dos xenocristais de granada apresentam-se bem conservadas, sendo minima a porcentagem de cristais quebrados. O mesmo se observa com o diamante, cuja porcentagem de fragmentos de clivagem é baixa. Esses fatos, associados à ocorrência periódica de grandes diamantes na região, só podem ser explicados admitindo-se a existência de kimberlitos mineralizados nas proximidades de Romaria.

Agradecimentos Os autores agradecem ao CNPq, à FAPESP e à FINEP pelo apoio financeiro para os trabalhos de campo, e ao Prof. Dr. Celso B. Gomes, do Instituto de Geociências da Universidade de São Paulo, pela leitura do manuscrito. 


\section{BIBLIOGRAFIA}

AKELLA, I, RAO, P.S, MCCALISTER, R.H, BOYD, F R , MEYER, $H$ O.A. - 1979 - Mineralogical studies on the diamondiferous kimberlite of the Wajrakliarur Area, Southern India. Proc. 2nd. International Kimberlite Conference (F.R. Boyd e H.O.A. Meyer, eds.), vol. 1, pp. 172-177. Amer. Geophys. Union, Washington, DC.

BARBOSA, O., BRAUN, O.P.G., DYER, R.C. e CUNHA, C.A.B.R. - $1970-$ Geologia do Triângulo Mineiro. Bol. n: 136, DNPM-DFPM, $140 \mathrm{pp}$.

BERGST $\emptyset$ L, S. - 1972 - The Jacupirangite at Kodal, Vestfold, Norway. Mine ral. Deposita 7: 233-246.

BOCT $\varnothing$ R, N.Z. c BOYD, F.R. - 1980 - Oxide minerals in the Liqhobong Kimberlite, Lesatho. Amer. Mineral. 65: 631-638.

BOCTOR, N.Z. C MEYER, H.O.A. - 1979-Oxide and Suffide minerals in Kimberlite from Green Mountain, Colorado. Proc. 2nd. International Kimberlite Conference (F,R. Boyd e H.O.A. Meyer, eds.), vol. 1, pp. 217-228. Amer. Geophys. Union, Washington, DC.

BOCTOR, N.Z. e SVISERO, D.P. - 1978 - Iron-titanium oxide and sulfide minerals in carbonatite from Jacupiranga, Brazil. Annual Rep. Direct. Geophys. Lab., Yearbook 77: 876-880, Washington, DC.

BOYD, F.R. E NIXON, P.H _.m-1973 - Origin of the ilmenite-silicate nodules in the kimbertices from Lesoltho and Africa. In: Lesotho Kimberites (P.H. Nixon, ed.), pp. 254-268. Lesotho Nat. Dev. Corp., Maseru, Lesotho.

CALÓGERAS, J.P. - 1895 - Contribution a l'étude des explorations de diamants at Brésil: Note sur les gisements diamantifères de Agua Suja. Rev. Univ. Mines Metalurgie XXIX: 1-21, Ljège; e Rev. Minas Gerais II 13: 5-8; 14: 33-37, Ouro Preto, MG.

CAMPOS, L.F.G. - 1891 - Jazidas Diamantíferas de Água Suja (Bagagem), Estado de Minas Gerais. Edit. Fluminense, 52 pp., Rio de Janeiro.

DRAPER, D. - $19 ! 1$ - The diamond-bearing deposits of Bagagem and Água Suja in State of Minas Gerais, Brazil. Trans. Geol Soc. South Africa 14: 8-19.

FERGUSON, J. e SHERATON, J.W. - 1979 - Petrogenesis of kimberlitic rocks and associated xenoliths of southeastern Australia. Proc. 2nd. International Kimberitite Conference (F.R. Boyd e H.O.A. Meyer, eds.), vol. 1, pp. 140-160. Amer. Geophys. Union, Washington, DC.

FRANTESESSON, E.V. - 1970 -. The Peirology of kimberlites. Publication no 150, Dept. of Geology, Australian University, $194 \mathrm{pp}$., Canberra.

FRICK, C. - 1973 - Kimberlitic ilmenites. Trans. Geol. Soc. South Africa 76 $85-94$.

GURNEY, J.J., FESQ, H.W. e KABLE, J.D. - 1973 - Clinopyroxene-itmenite intergrowths from kimberlites: A reappraisal: In: Lesotho Kimberlites (P.H. Nixon, ed.), pp. 238-253. Lesotho Nat. Dev. Corp., Maseru, Lesotho.

HAGGERTY, S.E. - 1975 - The chemistry and genesis of opaque minerals in kimberlites. Phys. Chem. of Earth 9: 295-307. Pergamon Press, New York.

HASUI, Y. - 1968 - A Formação Uberaba. An. XXII Congr, Bras, Geol., pp. 167.[79.

HASUI, Y, - 1969 - O Cretáceo no oeste mineiro, Bol. Soc, Bras, Geol. 18: 29-56.

HUSSAK, E. - 1891 - Noticia dos minerais das areias diamantiferas de Água Suja (Bagagem), Estado de Minas Gerais. In: Jazidas Diamantiferas de Água Suja (Bagagem), Estado de Minas Gerais (L.F.G. Campos, ed.), pp. 21-34, Rio de Janeiro.

HUSSAK, E. - 1894 - Sobre o depósito diamantífero de Água Suja perto de Bagagem, Minas Gerais. Relatório da Com. Explor. Planalto Central do Brasil, S: $281-319$, Rio de Janeiro.

LEONARDOS, O.H. - 1956 - Recursos minerais do Triângulo Mineiro. Rev. Min. Met. 140: 71-77; 141: 133-142; 142: 219-226.
LOVERING, J.F. c WIDDOWSON, J.R. - 1968 - Petrological environment of magnesium ifmenites. Earth Planet. Sci. Lett. 4: 310-314.

MCCALLISTER, R.H., MEYER, H.O.A. e BROOKINS, D.C. - 1975 - Pyroxene-ilmenite xenoliths from the Stockdale Pipe, Kansas: chemistry, crystallography and origin. Phys. Chem. of Earth 9: 287-293. Pergamon Press, New York.

MEYER, H.O.A. - 1977 - Mineralogy of the Upper Mantle: A review of the minerals in mantle xenoliths from kimberlites. Earth Sci. Rev. 13: 251-281.

MEYER, H.O.A. e SVISERO, D.P. - 1975 - Mineral inclusions in Brazilian diamonds. Phys. Chem. of Earth 9: 785-795. Pergamon Press, New York

MITCHELL., R.H. - 1973 - Magnesium ilmenite and its role in kimberlite petrogenesis. J. Geol. 81: 301-311.

MITCHELL, R.H. - 1979 - Mineralogy of the Tunraq Kimberlite, Somerset Is tand, N.W.T. Canada. Proc. 2nd. International Kimberlite Conference (F. R. Boyd e H.O.A. Meyer, eds.), vol. 1, pp. 161-171. Amer. Geophys. Union, Washington, DC.

NIXON, P.H. e KRESTEN, P. - 1973 - Chromium and nickel in kimberlitic itmenites. In: Lesotho Kimberlites (P.H. Nixon, ed.), pp. 235-237. Lesotho Nat. Dev, Corp., Maseru, Lesotho.

PASTERIS, J.D., BOYD, F.R. C NIXON, P.H. - 1979 - The itmenite association of the Frank Smith Mine, R.S.A. Proc. 2nd. International Kimberlite Conference (F.R. Boyd e H.O.A. Meyer, eds.), vol. 2, pp. 265-278. Amer. Geophys. Union, Washington, DC.

PORCHERON, H. - 1903 - Rapporl sur les mines de diamants de Água Suja dans l'Etal de Minas Gerais, Brésil. Lecoq et Mathorel, 18 pp., Paris.

SMITH, C.B., McCALLUM, M.E. C EGGLER, D.H. - 1976 - Clinopyroxeneitmenite intergrowths from the Iron Mountain Kimberlite District, Wyoming. Annual. Rep. Direct. Geophys. Lab., Yearbook 75: 542-544.

SOBOLEV, N.V. - 1977 - Deep-seated inclusions in kimberlites and the problem of the composition of the upper mantle (F.R. Byd, ed.), 279 pp., Amer. Geophys. Union, Washington, DC.

SOBOLEV, V.S., DOBRETSOV, N.L. e SOBOLEV, N.S. - 1975 - Xenólitos profundos e o Manto Superior. Editora Nauka, Novosibirsk, 269 pp. (em russo)

SUGUIO, K., SVISERO, D.P. e FELITTY, W. - 1979 - Conglomerados polimíticos cretáceos de Romaria. MG: Um exemplo de sedimentaçåo de leques aluviais, Atas 2. Simp. Reg. Geol. 1: 217-229.

SVISERO, D.P. - 1979 - Piropos cromiferos da mina de diamantes de Romaria, municipio de Romaria, MG. Bol. Mineral. 9: 7-14.

SVISERO, D.P., COIMBRA, A.M. e FEITOSA, V.M.N. - 1980 - Estudo mineralógico e químico dos concentrados da mina de diamantes de Romaria, municipio de Romaria, MG. An. XXXI Congr. Bras. Geol. 3: 1776-1788.

SVISERO, D.P., FELITTI, W. c ALMEIDA, J.S. - 1981 - Geologia da mina de diamantes de Romaria, municipio de Romaria, MG. Rev. Miner. Metal. 425: 4-14.

SVISERO, D.P., MEYER, H.O.A. e TSAI, H.M. - 1977 - Kimberlite minerals from Vargem (Minas Gerais) and Redondão (Piaui) diatremes, Brazil; and garnet therzolite xenolith from Redondão diatreme. Rev. Bras. Geoc. 7: 1-13.

SVISERO, D.P. MEYER, H.O.A. e TSAI, H.M - 1979 - Kimberlites in Brazil: An initial report. Proc. 2nd. International Kimberlite Conference (F.R. Boyd e H.O.A. Meyes, eds.), vol. 1, pp. 92-100. Amer. Geophys. Union, Washington, DC. 